\title{
Efeito da força iônica da solução de equilíbrio sobre a adsorção/dessorção de chumbo em Latossolos brasileiros ${ }^{(1)}$
}

\author{
Maria Aparecida Pereira Pierangeli(2), Luiz Roberto Guimarães Guilherme ${ }^{(3)}$, Leyser Rodrigues Oliveira(3), \\ Nilton Curi( ${ }^{(3)}$ e Marx Leandro Naves Silva(3)
}

\begin{abstract}
Resumo - A adsorção/dessorção de metais pelos solos é afetada por vários fatores, dentre os quais, a concentração salina da solução do solo. Este estudo teve o objetivo de avaliar o efeito da variação da força iônica da solução de equilíbrio $(I)$ sobre a adsorção/dessorção de $\mathrm{Pb}$ por Latossolos brasileiros. Amostras do horizonte A foram colocadas para reagir com $\mathrm{Pb}\left(\mathrm{NO}_{3}\right)_{2} 0,15 \mathrm{mmol} \mathrm{L}^{-1} \mathrm{em} \mathrm{Ca}\left(\mathrm{NO}_{3}\right)_{2} 5 \mathrm{e}$ $50 \mathrm{mmol} \mathrm{L}^{-1}\left(\mathrm{pH} 5,5 ; I=15\right.$ e $150 \mathrm{mmol} \mathrm{L}^{-1}$; relação solo:solução 1:100), durante 72 horas. Em seguida, as amostras foram colocadas para dessorver em $\mathrm{Ca}\left(\mathrm{NO}_{3}\right)_{2} 5 \mathrm{mmol} \mathrm{L}^{-1}$. Dos 17 Latossolos, apenas seis apresentaram diferenças significativas entre as quantidades adsorvidas nos dois valores de $I$. As correlações significativas obtidas entre as quantidades adsorvidas e alguns atributos dos solos $\left(\mathrm{SiO}_{2}\right.$ e $\mathrm{Fe}_{2} \mathrm{O}_{3}$ do ataque sulfúrico, $\mathrm{Fe}_{\mathrm{d}}$ e $\mathrm{Fe}_{\mathrm{o}}$, caulinita, hematita, CTC e superfície específica) foram as mesmas para os dois valores de $I$. A variação de $I$ durante a adsorção teve pouco efeito sobre a dessorção de chumbo. A não-variação da quantidade adsorvida com a mudança de $I$ indica que o $\mathrm{Pb}$ é adsorvido, na maioria dos Latossolos, como complexo de esfera interna. Conclui-se que, dentro destas condições experimentais, o $\mathrm{Pb}$ não fez parte do complexo de troca da maioria dos solos estudados.
\end{abstract}

Termos para indexação: metal pesado, íons, processo de transporte no solo, poluição do solo.

Effect of ionic strength of the equilibrium solution upon lead adsorption/desorption in Brazilian Oxisols

\begin{abstract}
The ionic strength of soil solution influences metal availability and mobility in soils. Laboratory experiments were conducted in order to evaluate the effect of solution ionic strength $(I)$ upon $\mathrm{Pb}$ adsorption/desorption in A-horizon samples of 17 Brazilian Oxisols. The effect of $I$ upon $\mathrm{Pb}$ adsorption was evaluated after a 72-hour reaction of the soil samples with $0.15 \mathrm{mmol} \mathrm{L}^{-1} \mathrm{~Pb}\left(\mathrm{NO}_{3}\right)_{2}$ at $\mathrm{pH} 5.5$, using 5 and $50 \mathrm{mmol} \mathrm{L}^{-1} \mathrm{Ca}\left(\mathrm{NO}_{3}\right)_{2}$ as background solutions $\left(I=15\right.$ and $\left.150 \mathrm{mmol} \mathrm{L}^{-1}\right)$. Lead desorption was measured after a 72-hour reaction of the soil samples with $5 \mathrm{mmol} \mathrm{L}^{-1} \mathrm{Ca}\left(\mathrm{NO}_{3}\right)_{2}$ at $\mathrm{pH} 5.5$. All experiments were performed with a 1:100 soil:solution ratio. Increasing $I$ caused $\mathrm{Pb}$ adsorption to decrease in six soils, while no significant change in $\mathrm{Pb}$ adsorption was observed for the remaining 11 Oxisols. Soil properties such as specific surface area, CEC, clay and hematite content, oxalate- and DCB-Fe, and sulfuric acid digestion-Si and -Fe correlated with $\mathrm{Pb}$ adsorption equally for both values of solution ionic strength. Changing $I$ had a small effect on $\mathrm{Pb}$ desorption. The little change in the amount of $\mathrm{Pb}$ adsorbed upon variation of $I$ indicates that $\mathrm{Pb}$ is adsorbed as an inner-sphere complex in most Oxisols. Therefore, this experiment suggests that $\mathrm{Pb}$ may not be part of the exchange complex of these soils.
\end{abstract}

Index terms: heavy metal, ions, soil transport processes, soil pollution.

(1)Aceito para publicação em 4 de outubro de 2000. Extraído da Dissertação de Mestrado apresentada pelo primeiro autor à Universidade Federal de Lavras (UFLA), Lavras, MG. Parcialmente financiado pela FAPEMIG.

(2)UFLA, Caixa Postal 37, CEP 37200-000 Lavras, MG. Bolsista da CAPES. E-mail: mapp@ufla.br

(3)UFLA. Bolsista do CNPq.

\section{Introdução}

$\mathrm{O} \mathrm{Pb}$ é um metal potencialmente tóxico para animais e plantas, e pode ser adicionado aos solos por meio de fertilizantes fosfatados, calcários, resíduos industriais, compostos orgânicos provenientes da reciclagem de lixo urbano e lodo de esgoto (Amaral 
Sobrinho et al., 1992; Kabata Pendias \& Pendias, 1992; Cravo et al., 1998). Sua biodisponibilidade, assim como a de outros cátions metálicos, é governada pela sua especiação na solução do solo e por suas interações com a fase sólida do sistema (Alloway, 1990). Metais pesados presentes em baixas concentrações tendem a ser retidos no solo via adsorção (McBride, 1994); este é o mais importante processo químico que afeta o comportamento e a biodisponibilidade de metais nos solos (Alloway, 1990).

A força iônica, que está relacionada à concentração total de eletrólitos, influencia a atividade dos íons em solução (Lindsay, 1979; McBride, 1994); é de se esperar que a adsorção de íons seja menor, em valores maiores de força iônica, devido à competição entre íons pelos sítios de troca e também pela diminuição de suas atividades. Entretanto, íons que são adsorvidos especificamente são menos influenciados pela variação da força iônica (Zelazny et al., 1996). Hu (1994), citado por Yu et al. (1997), trabalhando com solos de carga variável, concluiu que o aumento da força iônica diminuiu a adsorção de $\mathrm{Pb}$ e cobre. Por outro lado, Matos (1995), trabalhando com solos da região de Viçosa, $\mathrm{MG}$, observou que a adsorção de $\mathrm{Pb}$ e $\mathrm{Cu}$ foi pouco influenciada pela variação da concentração da solução de equilíbrio. Schindler et al. (1987) descrevem a retenção de $\mathrm{Pb}$ em caulinita como sendo tanto adsorção específica quanto adsorção não-específica, e concluíram que a troca de íons é importante a baixos valores de $\mathrm{pH}$ e força iônica, enquanto altos valores de $\mathrm{pH}$ e força iônica favorecem a adsorção específica.

De acordo com Naidu et al. (1994), a força iônica da solução do solo é menor que $0,005 \mathrm{~mol} \mathrm{~L}^{-1}$ nos solos dos trópicos, e maior que $0,10 \mathrm{~mol} \mathrm{~L}^{-1} \mathrm{em}$ solos menos intemperizados das regiões de clima temperado ou próximo a fertilizantes. Entretanto, ela pode ser alterada de várias maneiras, como resultado de adições ou perdas como pela adição de fertilizantes, lixiviação de cátions e ânions, absorção de nutrientes pelas plantas, deposições atmosféricas e outros.

Este trabalho objetivou verificar a influência da força iônica da solução de equilíbrio sobre a adsorção/ dessorção do $\mathrm{Pb}$ por Latossolos brasileiros.

\section{Material e Métodos}

O trabalho foi conduzido no Laboratório de Química e Mineralogia do Solo do Departamento de Ciência do Solo da Universidade Federal de Lavras (UFLA). A Tabela 1 apresenta a classificação e localização dos solos estudados e as Tabelas 2 e 3, os resultados das caracterizações química, física e mineralógica dos mesmos, realizadas conforme Silva et al. (1996).

Os valores de força iônica iniciais das soluções de equilíbrio de $\mathrm{Ca}\left(\mathrm{NO}_{3}\right)_{2}$ a 5 e $50 \mathrm{mmol} \mathrm{L}^{-1}$ foram 15 e $150 \mathrm{mmol} \mathrm{L}^{-1}$, respectivamente, calculadas de acordo com a seguinte equação, proposta por Debye-Hückel (Lindsay, 1979):

$I=1 / 2\left\{\sum_{\mathrm{i}} \mathrm{C}_{\mathrm{i}}\left(\mathrm{Z}_{\mathrm{i}}\right)^{2}\right\}$,

onde $I$ é a força iônica da solução, em mol L ${ }^{-1}$; C é a concentração, em mol L ${ }^{-1}$, dos íons presentes na solução, e $\mathrm{Z}$ refere-se às cargas ou valências desses íons. Os cálculos da força iônica final, das atividades de $\mathrm{Pb}^{2+} \mathrm{e} \mathrm{Ca}^{2+} \mathrm{e}$ a especiação de $\mathrm{Pb}^{2+}$ nas diferentes soluções contendo $\mathrm{Pb}$, usadas no experimento, foram realizados segundo o programa MINTEQA2 (Allison et al., 1990) (Tabela 4). Depois de preparadas, as soluções foram submetidas à aferição das forças iônicas, através de medidas das suas condutividades elétricas (Tabela 5). Nesse caso, a força iônica foi estimada pela relação (Griffin \& Jurinak, 1973, citados por Lindsay, 1979):

$I=0,013 \mathrm{CE}$, onde CE é a condutividade elétrica da solução em dS m $\mathrm{m}^{-1}$, e $I$ é a força iônica, em $\mathrm{mol} \mathrm{L}^{-1}$.

Nos cálculos de atividade iônica, por meio do programa MINTEQA2, optou-se pela utilização da equação de Debye-Hückel, na sua forma estendida (Lindsay, 1979), para a determinação dos coeficientes de atividade $(\gamma)$ dos íons $\mathrm{Pb}^{2+} \mathrm{e} \mathrm{Ca}^{2+}$ em solução:

$\log \gamma=-\mathrm{AZ}_{\mathrm{i}}^{2}\left\{I^{1 / 2} /\left(1+\mathrm{bd}_{\mathrm{i}} \mathrm{I}^{1 / 2}\right)\right\}$,

onde $\gamma$ é o coeficiente de atividade do íon; A é constante (de água a $\left.25^{\circ} \mathrm{C}=0,509\right) ; \mathrm{d}_{\mathrm{i}}$ é o tamanho do íon hidratado, em angstrons $\left(\mathrm{Pb}^{2+}=4,5 \AA \mathrm{e} \mathrm{Ca}^{2+}=6,0 \AA\right)$; b é igual a 0,33 , e os demais termos já foram definidos anteriormente.

Para medição da adsorção, amostras $(0,3 \mathrm{~g})$ da fração terra fina secada ao ar, em triplicata, foram pesadas em recipientes de vidros com capacidade de $50 \mathrm{~mL}$ e colocadas em suspensão com $20 \mathrm{~mL}$ de $\mathrm{Ca}\left(\mathrm{NO}_{3}\right)_{2} 5 \mathrm{mmol} \mathrm{L}^{-1} \mathrm{e}$ $\mathrm{Ca}\left(\mathrm{NO}_{3}\right)_{2} 50 \mathrm{mmol} \mathrm{L}^{-1}$ (relação solo:solução inicial 1:67), para as forças iônicas de $15 \mathrm{mmol} \mathrm{L}^{-1}$ e $150 \mathrm{mmol} \mathrm{L}^{-1}$, respectivamente. Em seguida, foram adicionadas quantidades adequadas de solução saturada de $\mathrm{Ca}(\mathrm{OH})_{2}$ para ajustar o $\mathrm{pH}$ a $5,5 \pm 0,1$. Após essa operação as soluções permaneceram por 72 horas, para que fosse atingido o $\mathrm{pH}$ de equilíbrio, alternando-se um período de 12 horas com agitação e 12 horas em repouso. Após este período, o qual foi 
Tabela 1. Classificação e localização dos solos estudados.

\begin{tabular}{|c|c|c|c|}
\hline Solo & Classificação & Localização & Material de origem \\
\hline 1 & $\begin{array}{l}\text { Latossolo Roxo distrófico textura argilosa fase floresta } \\
\text { subtropical }\end{array}$ & Ijuí, RS & Basalto \\
\hline 2 & $\begin{array}{l}\text { Latossolo Vermelho-Escuro distrófico textura argilosa } \\
\text { fase floresta subtropical }\end{array}$ & Passo Fundo, RS & Basalto e arenito (mistura) \\
\hline 3 & $\begin{array}{l}\text { Latossolo Roxo distrófico textura argilosa fase floresta } \\
\text { subtropical alta mista com araucária }\end{array}$ & Chapecó, SC & Basalto \\
\hline 4 & $\begin{array}{l}\text { Latossolo Vermelho-Escuro distrófico textura argilosa } \\
\text { fase floresta tropical perenifólia }\end{array}$ & Ponta Grossa, PR & $\begin{array}{l}\text { Rochas diversas do Pré- } \\
\text { cambriano }\end{array}$ \\
\hline 5 & $\begin{array}{l}\text { Latossolo Roxo eutrófico textura argilosa fase floresta } \\
\text { tropical subperenifólia }\end{array}$ & Londrina, PR & Basalto, meláfiro e diabásio \\
\hline 6 & $\begin{array}{l}\text { Latossolo Vermelho-Escuro álico textura média fase } \\
\text { floresta tropical subperenifólia }\end{array}$ & Paranavaí, PR & Arenito Caiuá \\
\hline 7 & $\begin{array}{l}\text { Latossolo Roxo eutrófico textura muito argilosa fase } \\
\text { floresta subperenifólia }\end{array}$ & Dourados, MS & Rochas eruptivas básicas \\
\hline 8 & $\begin{array}{l}\text { Latossolo Roxo eutrófico textura muito argilosa fase } \\
\text { floresta tropical subcaducifólia }\end{array}$ & Campinas, SP & Diabásio \\
\hline 9 & $\begin{array}{l}\text { Latossolo Vermelho-Escuro distrófico textura argilosa } \\
\text { fase floresta latifoliada tropical }\end{array}$ & Jaboticabal, SP & $\begin{array}{l}\text { Basalto, com influência de } \\
\text { arenito }\end{array}$ \\
\hline 10 & $\begin{array}{l}\text { Latossolo Vermelho-Escuro distrófico textura muito } \\
\text { argilosa fase cerrado }\end{array}$ & Lavras, MG & Gnaisse granítico mesocrático \\
\hline 11 & $\begin{array}{l}\text { Latossolo Vermelho-Amarelo distrófico } \\
\text { textura muito argilosa fase cerrado }\end{array}$ & Lavras, MG & Gnaisse granítico leucocrático \\
\hline 12 & $\begin{array}{l}\text { Latossolo Vermelho-Escuro álico textura muito } \\
\text { argilosa fase cerrado tropical subcaducifólio }\end{array}$ & Sete Lagoas, MG & $\begin{array}{l}\text { Rochas pelíticas do Grupo } \\
\text { Bambuí }\end{array}$ \\
\hline 13 & $\begin{array}{l}\text { Latossolo Vermelho-Escuro distrófico textura argilosa } \\
\text { fase cerrado tropical subcaducifólio }\end{array}$ & Goiânia, GO & $\begin{array}{l}\text { Sedimentos argilosos } \\
\text { retrabalhados }\end{array}$ \\
\hline 14 & $\begin{array}{l}\text { Latossolo Vermelho-Escuro álico textura argilosa fase } \\
\text { cerradão subcaducifólio }\end{array}$ & Planaltina, DF & $\begin{array}{l}\text { Sedimentos argilosos de } \\
\text { cobertura }\end{array}$ \\
\hline 15 & $\begin{array}{l}\text { Latossolo Vermelho-Amarelo álico textura média fase } \\
\text { floresta subperenifólia }\end{array}$ & Areia, PB & Sedimentos areno-argilosos \\
\hline 16 & $\begin{array}{l}\text { Latossolo Vermelho-Amarelo distrófico textura média } \\
\text { fase floresta subperenifólia }\end{array}$ & Ubajara, CE & Arenito \\
\hline 17 & $\begin{array}{l}\text { Latossolo Amarelo álico textura argilosa fase floresta } \\
\text { equatorial subperenifólia }\end{array}$ & Tomé Açu, PA & Sedimentos do Terciário \\
\hline
\end{tabular}

estabelecido com base no estudo de Guilherme \& Anderson (1998), foi medido o pH das soluções e foram adicionados, em cada vidro, $10 \mathrm{~mL}$ de solução de $\mathrm{Pb}\left(\mathrm{NO}_{3}\right)_{2}$ $0,45 \mathrm{mmol} \mathrm{L}^{-1}$ com a força iônica ajustada de acordo com cada experimento (relação solo:solução final 1:100; concentração final de $\left.\mathrm{Pb}=0,15 \mathrm{mmol} \mathrm{L}^{-1}\right)$. As amostras foram, então, deixadas para reagir por 72 horas, alternandose 12 horas de repouso e 12 horas de agitação, em agitador horizontal. Findo este período, elas foram centrifugadas; 0 sobrenadante coletado para leitura de $\mathrm{Pb}$; e o resíduo pesado, para determinação da massa de solução retida. A quantidade adsorvida foi calculada pela diferença entre $\mathrm{o} \mathrm{Pb}$ adicionado e o remanescente no sobrenadante. Uma concentração de $\mathrm{Pb}$ de $0,15 \mathrm{mmol} \mathrm{L}^{-1}$ foi usada para evitar a excessiva formação de pares iônicos, ou até mesmo a precipitação de $\mathrm{Pb}$ em solução (Allison et al., 1990).

Ao resíduo remanescente do experimento de adsorção adicionaram-se $30 \mathrm{~mL}$ de solução de $\mathrm{Ca}\left(\mathrm{NO}_{3}\right)_{2} 5 \mathrm{mmol} \mathrm{L}^{-1}$ com $\mathrm{pH}$ ajustado para 5,5, para realização da dessorção do $\mathrm{Pb}$ retido nas amostras de solo; repetiu-se o mesmo procedimento com relação ao período de agitação e repouso realizado durante a adsorção. Após 72 horas, as amostras foram centrifugadas e o sobrenadante, coletado, para leitura. A quantidade dessorvida foi calculada, descontando-se a quantidade presente na massa de solução retida após a adsorção. 
Tabela 2. Atributos físicos, químicos e mineralógicos e relações destes atributos em Latossolos brasileiros ${ }^{(1)}$.

\begin{tabular}{|c|c|c|c|c|c|c|c|c|c|c|c|c|c|c|c|c|}
\hline \multirow[t]{2}{*}{ Solo } & \multicolumn{4}{|c|}{ Mineralogia } & \multicolumn{3}{|c|}{ Ataque sulfúrico } & \multirow[t]{2}{*}{$\mathrm{Ki}$} & \multirow[t]{2}{*}{$\mathrm{Kr}$} & \multicolumn{2}{|c|}{$\mathrm{Fe}_{2} \mathrm{O}_{3}$} & \multirow[t]{2}{*}{$\mathrm{Fe}_{\mathrm{d}} / \mathrm{Fe}_{\mathrm{s}}$} & \multirow[t]{2}{*}{$\mathrm{Fe}_{\mathrm{o}} / \mathrm{Fe}_{\mathrm{d}}$} & \multirow[t]{2}{*}{$\mathrm{T}$} & \multirow[t]{2}{*}{$\mathrm{SE}$} & \multirow[t]{2}{*}{$\mathrm{MO}$} \\
\hline & $\mathrm{Ct}$ & $\mathrm{Gb}$ & $\mathrm{Gt}$ & $\mathrm{Hm}$ & $\mathrm{SiO}_{2}$ & $\mathrm{Al}_{2} \mathrm{O}_{3}$ & $\mathrm{Fe}_{2} \mathrm{O}_{3}$ & & & $\mathrm{DCB}$ & $\mathrm{Ox}$ & & & & & \\
\hline & $-\cdots$ & - & $\ldots$ & (g kg & )----. & & & & & $---(g$ & 1) ---- & & & $\left(\mathrm{mmol}_{\mathrm{c}} \mathrm{kg}^{-1}\right)$ & $\left(m^{2} g^{-1}\right)$ & $\left(\mathrm{g} \mathrm{kg}^{-1}\right)$ \\
\hline 1 & 290 & 40 & 7 & 74 & 199 & 182 & 210 & 1,9 & 0,9 & 80 & 3,7 & 0,38 & 0,05 & 148 & 187,6 & 26 \\
\hline 2 & 80 & 30 & 2 & 28 & 149 & 143 & 63 & 1,8 & 1,2 & 38 & 2,8 & 0,60 & 0,07 & 181 & 139,7 & 31 \\
\hline 3 & 430 & 50 & 3 & 58 & 246 & 217 & 139 & 1,9 & 1,2 & 62 & 2,7 & 0,44 & 0,08 & 158 & 196,6 & 45 \\
\hline 4 & 220 & 110 & 27 & 37 & 172 & 220 & 178 & 1,3 & 0,7 & 87 & 2,5 & 0,49 & 0,03 & 79 & 166,1 & 22 \\
\hline 5 & 280 & 150 & 7 & 81 & 253 & 270 & 268 & 1,6 & 0,8 & 119 & 6,0 & 0,44 & 0,05 & 104 & 182,3 & 14 \\
\hline 6 & 90 & 10 & 2 & 8 & 42 & 66 & 27 & 1,1 & 0,8 & 11 & 0,7 & 0,41 & 0,06 & 41 & 90,4 & 9 \\
\hline 7 & 250 & 70 & 7 & 49 & 203 & 213 & 301 & 1,6 & 0,7 & 76 & 3,2 & 0,25 & 0,04 & 117 & 169,9 & 13 \\
\hline 8 & 190 & 110 & 21 & 37 & 161 & 208 & 178 & 1,3 & 0,7 & 78 & 3,0 & 0,44 & 0,04 & 86 & 166,6 & 26 \\
\hline 9 & 170 & 140 & 3 & 15 & 114 & 192 & 100 & 1,0 & 0,7 & 51 & 2,1 & 0,51 & 0,04 & 66 & 147,0 & 29 \\
\hline 10 & 190 & 530 & 16 & 19 & 159 & 270 & 125 & 1,0 & 0,7 & 46 & 2,2 & 0,37 & 0,05 & 60 & 156,1 & 33 \\
\hline 11 & 220 & 270 & 16 & 30 & 141 & 330 & 110 & 0,7 & 0,5 & 60 & 0,3 & 0,54 & 0,05 & 64 & 182,6 & 48 \\
\hline 12 & 310 & 160 & 9 & 46 & 234 & 319 & 120 & 1,2 & 0,9 & 73 & 3,4 & 0,61 & 0,05 & 41 & 171,6 & 38 \\
\hline 13 & 10 & 240 & 14 & 24 & 95 & 241 & 108 & 0,7 & 0,5 & 59 & 2,2 & 0,55 & 0,04 & 74 & 145,3 & 30 \\
\hline 14 & 240 & 50 & 11 & 19 & 150 & 187 & 88 & 1,4 & 0,9 & 52 & 3,1 & 0,59 & 0,06 & 103 & 159,8 & 41 \\
\hline 15 & 290 & 20 & 2 & 5 & 135 & 149 & 39 & 1,5 & 1,2 & 15 & 1,8 & 0,38 & 0,12 & 110 & 162,9 & 43 \\
\hline 16 & 20 & 20 & 3 & 0 & 46 & 75 & 15 & 1,0 & 0,9 & 9 & 0,9 & 0,60 & 0,10 & 88 & 91,0 & 26 \\
\hline 17 & 260 & 20 & 5 & 0 & 124 & 135 & 33 & 1,6 & 1,2 & 10 & 0,7 & 0,30 & 0,07 & 88 & 103,1 & 16 \\
\hline
\end{tabular}

${ }^{(1)} \mathrm{Ct}$ : caulinita; Gb: gibbsita; Gt: goethita; $\mathrm{Hm}$ : hematita; Ki: relação molecular $\mathrm{SiO}_{2} / \mathrm{Al}_{2} \mathrm{O}_{3} ; \mathrm{Kr}$ : relação molecular $\mathrm{SiO}_{2} /\left(\mathrm{Al}_{2} \mathrm{O}_{3}+\mathrm{Fe}_{2} \mathrm{O}_{3}\right) ; \mathrm{Fe}_{\mathrm{d}}$ : $\mathrm{Fe}_{2} \mathrm{O}_{3}$ extraído pelo ditionito citrato bicarbonato; $\mathrm{Fe}_{\mathrm{o}}: \mathrm{Fe}_{2} \mathrm{O}_{3}$ extraído pelo oxalato de amônio; $\mathrm{Fe}_{\mathrm{s}}$ : $\mathrm{Fe}_{2} \mathrm{O}_{3}$ extraído pelo ataque sulfúrico; T: CTC a pH 7,0 $\left(\mathrm{mmol}_{\mathrm{c}} \mathrm{kg}^{-1}\right)$; SE: superfície específica; MO: matéria orgânica.

Tabela 3. Granulometria e relações de atributos em Latossolos brasileiros ${ }^{(1)}$.

\begin{tabular}{|c|c|c|c|c|c|}
\hline \multirow[t]{2}{*}{ Solo } & \multicolumn{3}{|c|}{ Granulometria $\left(\mathrm{g} \mathrm{kg}^{-1}\right)$} & \multicolumn{2}{|c|}{ Relações } \\
\hline & Areia & Silte & Argila & $\mathrm{Gb} /(\mathrm{Gb}+\mathrm{Ct})$ & $\mathrm{Gt} /(\mathrm{Gt}+\mathrm{Hm})$ \\
\hline 1 & 170 & 160 & 670 & 0,12 & 0,09 \\
\hline 2 & 470 & 60 & 470 & 0,27 & 0,07 \\
\hline 3 & 60 & 150 & 790 & 0,10 & 0,05 \\
\hline 4 & 290 & 80 & 630 & 0,33 & 0,42 \\
\hline 5 & 60 & 80 & 860 & 0,35 & 0,08 \\
\hline 6 & 860 & 1 & 139 & 0,10 & 0,20 \\
\hline 7 & 240 & 110 & 650 & 0,22 & 0,12 \\
\hline 8 & 300 & 100 & 600 & 0,37 & 0,36 \\
\hline 9 & 660 & 1 & 339 & 0,45 & 0,17 \\
\hline 10 & 180 & 100 & 720 & 0,74 & 0,46 \\
\hline 11 & 170 & 110 & 720 & 0,55 & 0,35 \\
\hline 12 & 120 & 30 & 850 & 0,34 & 0,16 \\
\hline 13 & 360 & 100 & 540 & 0,96 & 0,37 \\
\hline 14 & 370 & 110 & 520 & 0,17 & 0,37 \\
\hline 15 & 550 & 10 & 440 & 0,06 & 0,28 \\
\hline 16 & 800 & 50 & 150 & 0,50 & 1,00 \\
\hline 17 & 600 & 40 & 360 & 0,07 & 1,00 \\
\hline
\end{tabular}

O delineamento experimental foi inteiramente casualizado, em esquema fatorial $17 \times 2$, sendo 17 solos, dois valores de força iônica, e três repetições. Os dados obtidos foram submetidos à análise estatística e a influência dos atributos químicos, físicos e mineralógicos dos solos sobre a adsorção/dessorção de $\mathrm{Pb}$, nos dois valores de força iônica, foi avaliada por meio de análise de correlação simples.

\section{Resultados e Discussão}

Apenas seis solos apresentaram diferenças significativas entre as quantidades adsorvidas nas duas forças iônicas, com maiores quantidades no menor valor de força iônica (Figura 1). O efeito da força iônica (I) sobre a adsorção de metais tem sido atribuído a: 1) mudança na atividade dos íons livres (p. ex., $\mathrm{Pb}^{2+}, \mathrm{Cd}^{2+}$ e $\mathrm{Zn}^{2+}$ ) por causa da formação de pares iônicos, e do efeito de $I$ sobre o pH (Davis \& Leckie, 1978); 2) competição pelos sítios de adsorção entre o metal e os eletrólitos presentes no meio (Spark et al., 1995); 3) mudanças do potencial eletrostático no plano de adsorção (Barrow, 1986). Como, neste estudo, o pH das soluções e das suspensões com solo foi ajustado para 5,5, o efeito de $I$ sobre o $\mathrm{pH}$ é pouco provável. $\mathrm{O}$ aumento da força iônica da solução de equilíbrio $\left(\mathrm{Ca}\left(\mathrm{NO}_{3}\right)_{2}\right)$ de 15 para $150 \mathrm{mmol} \mathrm{L}^{-1}$ resultou no aumento da concentração do par iônico $\mathrm{PbNO}_{3}{ }^{+}$, com redução na atividade de $\mathrm{Pb}^{2+}$ de cerca 2,5 vezes, ao passo que a atividade do $\mathrm{Ca}^{2+}$ aumentou 6,0 vezes (Tabela 4). Apenas este fato já justificaria uma redução na quantidade de $\mathrm{Pb}$ adsorvida quando se elevou $I$, pois, segundo Harter (1979), o cálcio é um forte competidor com $\mathrm{Pb}$ pelos sítios de adsorção. Ressalta-se, entretanto, que o trabalho de Harter (1979) não foi realizado em solos com predomínio de carga variável, como os Latossolos. 
Supõe-se que nos solos onde diferenças significativas foram detectadas $\mathrm{o} \mathrm{Pb}$ esteja adsorvido preferencialmente de forma não-específica, uma vez que o decréscimo da adsorção e o aumento da concentração do meio são tipicamente atribuídos ao efeito da força iônica e interpretados como evidências de formação de complexos de esfera externa (Hayes \& Leckie, 1986; Zelazny et al., 1996). Já nos solos que não apresentaram diferenças significativas nas quantidades adsorvidas, supõe-se que o $\mathrm{Pb}$ seja adsorvido mais especificamente, uma vez que a variação de $I$ não afetou a quantidade adsorvida (Barrow, 1989; Zelazny et al., 1996), o que indica maior afinidade do metal com os constituintes do solo, ou seja, a formação de complexos de esfera interna.

As quantidades adsorvidas apresentaram as mesmas correlações significativas com alguns atributos dos solos, nos dois valores de $I$ (Tabela 6), ou seja,

Tabela 4. Especiação de $\mathrm{Pb}^{2+}$ a pH 5,5, força iônica final $\left(I_{\text {final }}\right)$ e atividades de $\mathrm{Pb}^{2+}\left(\mathrm{a}_{\mathrm{Pb}}\right)$ e $\mathrm{Ca}^{2+}\left(\mathrm{a}_{\mathrm{Ca}}\right)$ em soluções de $\mathrm{Pb}\left(\mathrm{NO}_{3}\right)_{2}$ 0,15 mmol L-1, empregando-se duas soluções de equilíbrio de $\mathrm{Ca}\left(\mathrm{NO}_{3}\right)_{2}\left(5\right.$ e $\left.50 \mathrm{mmol} \mathrm{L}^{-1}\right)$. $\mathrm{P}_{\mathrm{CO}_{2}}=0,03 \mathrm{kPa}(0,0003 \mathrm{~atm})^{(1)}$.

\begin{tabular}{ccc}
\hline Parâmetro calculado & \multicolumn{2}{c}{ Solução de equilíbrio } \\
\cline { 2 - 3 } & $\begin{array}{cc}\mathrm{Ca}\left(\mathrm{NO}_{3}\right)_{2} 5 \mathrm{mmol} \mathrm{L}^{-1} \\
\left(I=15 \mathrm{mmol} \mathrm{L}^{-1}\right)\end{array}$ & $\begin{array}{c}\mathrm{Ca}\left(\mathrm{NO}_{3}\right)_{2} 50 \mathrm{mmol} \mathrm{L}^{-1} \\
\left(I=150 \mathrm{mmol} \mathrm{L}^{-1}\right)\end{array}$ \\
\hline Espécies de $\mathrm{Pb}(\%)$ & & \\
$\mathrm{Pb}^{2+}$ & 91,20 & 68,70 \\
$\mathrm{PbNO}_{3}^{+}$ & 8,40 & 31,10 \\
$\mathrm{PbOH}^{+}$ & 0,40 & 0,20 \\
\hline$I_{\text {final }}\left(\mathrm{mmol} \mathrm{L}^{-1}\right)$ & 15,43 & 150,400 \\
$\mathrm{a}_{\mathrm{Ca}}\left(\mathrm{mmol} \mathrm{L}^{-1}\right)$ & 3,145 & 18,830 \\
$\mathrm{a}_{\mathrm{Pb}}\left(\mathrm{mmol} \mathrm{L}^{-1}\right)$ & 0,083 & 0,033 \\
\hline${ }^{(1)} \mathrm{Cálculos} \mathrm{efetuados} \mathrm{através} \mathrm{do} \mathrm{programa} \mathrm{MINTEQA2} \mathrm{(Allison}$ \\
et al., 1990).
\end{tabular}

Tabela 5. Valores de condutividade elétrica (CE) e força iônica estimada $\left(I_{\text {est }}\right)$ das soluções usadas no experimento.

\begin{tabular}{lcc}
\hline Solução & $\mathrm{CE}\left(\mathrm{dS} \mathrm{m}{ }^{-1}\right)$ & $I_{\text {est }}\left(\mathrm{mmol} \mathrm{L}^{-1}\right)^{(1)}$ \\
\hline $\mathrm{Ca}\left(\mathrm{NO}_{3}\right)_{2} 5 \mathrm{mmol} \mathrm{L}^{-1}$ & 1,042 & 13,55 \\
$\mathrm{Ca}\left(\mathrm{NO}_{3}\right)_{2} 50 \mathrm{mmol} \mathrm{L}^{-1}$ & 8,740 & 113,62 \\
$\mathrm{~Pb}\left(\mathrm{NO}_{3}\right)_{2} 0,15 \mathrm{mmol} \mathrm{L}^{-1} \mathrm{em}$ & & \\
$\mathrm{Ca}\left(\mathrm{NO}_{3}\right)_{2} 5 \mathrm{mmol} \mathrm{L}^{-1}$ & 1,425 & 18,53 \\
$\mathrm{~Pb}\left(\mathrm{NO}_{3}\right)_{2} 0,15 \mathrm{mmol} \mathrm{L}^{-1} \mathrm{em}$ & & \\
$\mathrm{Ca}\left(\mathrm{NO}_{3}\right)_{2} 50 \mathrm{mmol} \mathrm{L}^{-1}$ & 8,350 & 108,55 \\
\hline${ }^{(1)} I_{\text {est }}$ força iônica estimada a partir da equação $I\left(\mathrm{~mol} \mathrm{~L}^{-1}\right)=0,013 \mathrm{CE}\left(\mathrm{dS} \mathrm{m}^{-1}\right)$.
\end{tabular}

as diferenças na adsorção de $\mathrm{Pb}$, significativas ou não, causadas pela variação da força iônica, não foram suficientes para alterar o efeito dos atributos do solo avaliados sobre a adsorção de chumbo. A correlação significativa positiva obtida com $\mathrm{Fe}_{2} \mathrm{O}_{3}$ e $\mathrm{Fe}_{\mathrm{o}}$ demonstra a afinidade dos óxidos de $\mathrm{Fe}$ por $\mathrm{Pb}$, principalmente a hematita, conforme já relatado por McKenzie (1980). Este fato, não apenas com relação a $\mathrm{Pb}$, tem sido amplamente documentado em literatura (Matos et al., 1996; Axe \& Anderson, 1998). Entretanto, os seis solos que apresentaram diferenças significativas entre as quantidades adsorvidas, nos dois valores de $I$, apresentam altos teores de $\mathrm{Fe}$ (Latossolo Roxo e Latossolo Vermelho-Escuro), o que indica que o efeito da força iônica sobre a adsorção de cátions metálicos, mesmo sobre substratos semelhantes quimicamente, pode diferir significativamente (Spark et al., 1995).

Não houve efeito da força iônica sobre a dessorção de $\mathrm{Pb}$, havendo diferenças significativas

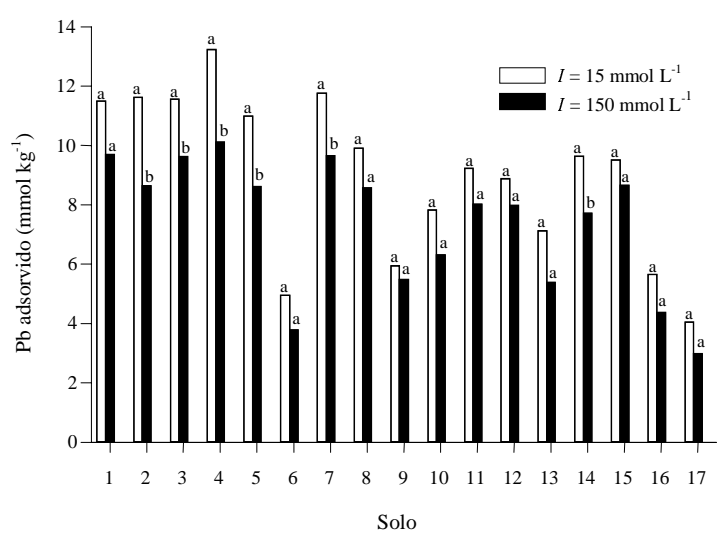

Figura 1. Chumbo adsorvido por amostras de horizonte A de 17 Latossolos provenientes de todas as regiões geográficas brasileiras, em dois valores de força iônica $(I=15$ e $\left.150 \mathrm{mmol} \mathrm{L}^{-1}\right)$ da solução de equilíbrio $\left(\mathrm{Ca}\left(\mathrm{NO}_{3}\right)_{2}\right)$. (Concentração inicial de $\mathrm{Pb}=0,15 \mathrm{mmol} \mathrm{L}^{-1}$; $\mathrm{pH} 5,5$; relação solo:solução 1:100. Barras seguidas de mesma letra, dentro do mesmo solo, não diferem entre si pelo teste de Tukey a 5\%. Os solos estão apresentados na Tabela 1.) 
apenas entre os solos. Além disso, a fração de $\mathrm{Pb}$ dessorvida $(\mathrm{Pb}$ dessorvido/ $\mathrm{Pb}$ adsorvido) decresceu de maneira semelhante nos dois valores de $I$ (Figura 2). Em média, da quantidade total de $\mathrm{Pb}$ adsorvido, $74 \%$ ainda permaneceu retida nos solos após 72 horas de realização da dessorção, para $I=150 \mathrm{mmol} \mathrm{L}^{-1}$, e $77 \%$, para $I=15 \mathrm{mmol} \mathrm{L}^{-1}$; isto indica que a solução de $\mathrm{Ca}\left(\mathrm{NO}_{3}\right)_{2} 5 \mathrm{mmol} \mathrm{L}^{-1}$ foi pouco eficiente para dessorver o $\mathrm{Pb}$ adsorvido nos solos, ou que a maior parte do $\mathrm{Pb}$ ficou retido por meio de ligações não eletrostáticas. Estes resultados são corroborados pelos de Chlopecka et al. (1996) trabalhando com solos da Polônia, e Matos et al. (1996) trabalhando com Latossolo Vermelho-Amarelo da região de Viçosa, MG, os quais verificaram que muito pouco $\mathrm{Pb}$ foi retido na fase trocável.

Tabela 6. Coeficientes de correlação de Pearson (r) entre alguns atributos dos solos e as quantidades de $\mathrm{Pb}$ adsorvidas, em duas forças iônicas (I) da solução de equilíbrio.

\begin{tabular}{|c|c|c|}
\hline Atributo $^{(1)}$ & $I=15 \mathrm{mmol} \mathrm{L}^{-1}$ & $I=150 \mathrm{mmol} \mathrm{L}^{-1}$ \\
\hline $\mathrm{MO}$ & 0,27 & 0,26 \\
\hline SE & $0,80 * *$ & $0,83 * *$ \\
\hline Caulinita $(\mathrm{Ct})$ & $0,46^{*}$ & $0,54 *$ \\
\hline Gibbsita (Gb) & $-0,03$ & $-0,07$ \\
\hline Goethita (Gt) & 0,34 & 0,29 \\
\hline Hematita (Hm) & $0,70 * *$ & $0,72 * *$ \\
\hline $\mathrm{Gt} /(\mathrm{Gt}+\mathrm{Hm})$ & $-0,56^{*}$ & $-0,68 * *$ \\
\hline $\mathrm{Gb} /(\mathrm{Gb}+\mathrm{Ct})$ & $-0,16$ & $-0,26$ \\
\hline $\mathrm{Fe}_{2} \mathrm{O}_{3}$ & $0,68 * *$ & $0,66^{* *}$ \\
\hline $\mathrm{Al}_{2} \mathrm{O}_{3}$ & 0,43 & 0,41 \\
\hline $\mathrm{SiO}_{2}$ & $0,74 * *$ & $0,73 * *$ \\
\hline Silte & $0,60 *$ & $0,53 *$ \\
\hline Argila & $0,57 *$ & $0,54 *$ \\
\hline $\mathrm{Ki}$ & $0,53 *$ & $0,52 *$ \\
\hline $\mathrm{Kr}$ & 0,22 & 0,16 \\
\hline $\mathrm{Al}_{2} \mathrm{O}_{3} / \mathrm{Fe}_{2} \mathrm{O}_{3}$ & $-0,64 * *$ & $-0,67 * *$ \\
\hline CTC a pH 7,0 (T) & $0,59 *$ & $0,49 *$ \\
\hline $\mathrm{Fe}_{\mathrm{d}}$ & $0,70 * *$ & $0,67 * *$ \\
\hline $\mathrm{Fe}_{\mathrm{o}}$ & $0,66^{* *}$ & $0,63 * *$ \\
\hline $\mathrm{Fe}_{\mathrm{o}} / \mathrm{Fe}_{\mathrm{d}}$ & $-0,20$ & $-0,20$ \\
\hline $\mathrm{Fe}_{\mathrm{d}} / \mathrm{Fe}_{\mathrm{s}}$ & 0,05 & $-0,04$ \\
\hline
\end{tabular}

(1) $\mathrm{SE}$ : superfície específica; $\mathrm{MO}$ : matéria orgânica; $\mathrm{Fe}_{2} \mathrm{O}_{3}, \mathrm{Al}_{2} \mathrm{O}_{3}$ $\mathrm{SiO}_{2}$ : óxidos totais extraídos pelo ataque sulfúrico; Ki: relação molecular $\mathrm{SiO}_{2} / \mathrm{Al}_{2} \mathrm{O}_{3}$; Kr: relação molecular $\mathrm{SiO}_{2} /\left(\mathrm{Al}_{2} \mathrm{O}_{3}+\mathrm{Fe}_{2} \mathrm{O}_{3}\right) ; \mathrm{Fe}_{\mathrm{d}}: \mathrm{Fe}_{2} \mathrm{O}$ extraído pelo ditionito citrato bicarbonato; $\mathrm{Fe}_{\mathrm{o}}: \mathrm{Fe}_{2} \mathrm{O}_{3}$ extraído pelo oxalato de amônio; $\mathrm{Fe}_{\mathrm{s}}: \mathrm{Fe}_{2} \mathrm{O}_{3}$ extraído pelo ataque sulfúrico. ${ }^{*} \mathrm{e} *{ }^{*} \mathrm{Sig}$ nificativo a $5 \%$ e a $1 \%$ de probabilidade, respectivamente, pelo teste $\mathrm{F}$.

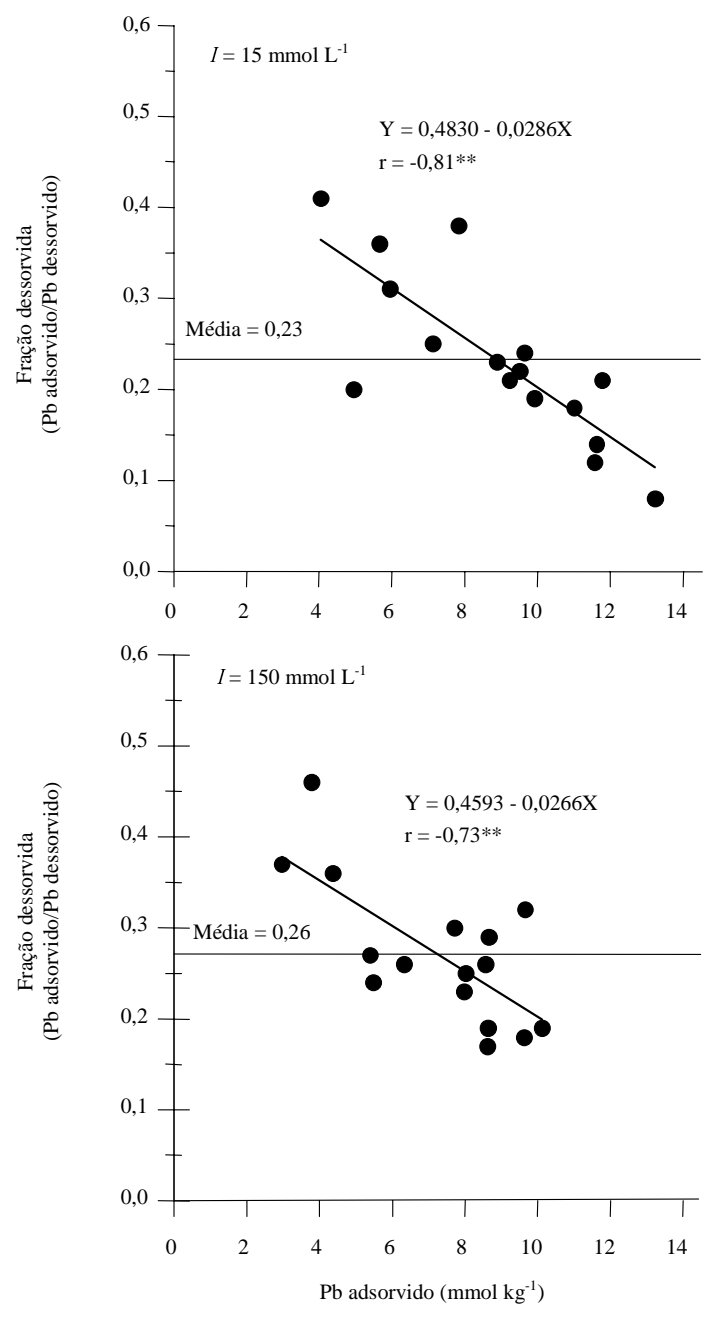

Figura 2. Relação entre $\mathrm{Pb}$ adsorvido, em dois valores de força iônica $\left(I=15\right.$ e $\left.150 \mathrm{mmol} \mathrm{L}^{-1}\right)$ da solução de equilíbrio $\left(\mathrm{Ca}\left(\mathrm{NO}_{3}\right)_{2}\right)$, e fração de $\mathrm{Pb}$ dessorvida ( $\mathrm{Pb}$ dessorvido/ $\mathrm{Pb}$ adsorvido) em $\mathrm{Ca}\left(\mathrm{NO}_{3}\right)_{2} 5 \mathrm{mmol} \mathrm{L}^{-1}$ por amostras de horizonte A de 17 Latossolos provenientes de todas as regiões geográficas brasileiras. (Concentração inicial de $\mathrm{Pb}=0,15 \mathrm{mmol} \mathrm{L}^{-1} ; \mathrm{pH} 5,5$; relação solo:solução 1:100.)

\section{Conclusões}

1. A não-variação da quantidade adsorvida com a mudança da força iônica da solução de equilíbrio indica que, na maioria dos Latossolos estudados, o $\mathrm{Pb}$ é adsorvido predominantemente como complexo de esfera interna. 
2. Evidências de adsorção específica sugerem que o $\mathrm{Pb}$ não faz parte do complexo de troca da maioria dos solos estudados, e pode ser substituído apenas por outras espécies de cátions com maior afinidade para com seus constituintes.

\section{Referências}

ALLISON, J. D.; BROWN, D. S.; NOVO-GRADAC, K. J. MINTEQA2/PRODEFA2: a geochemical assessment model for environmental systems: version 3.00 user's manual. Athens : United States Environmental Protection Agency, 1990. 106 p. (EPA-600/3-91-021).

ALLOWAY, B. J. Heavy metals in soils. New York : J. Wiley, 1990. $339 \mathrm{p}$.

AMARAL SOBRINHO, N. M. B.; COSTA, L. M.; OLIVEIRA, C.; VELOSO, A. C. X. Metais pesados em alguns solos e corretivos. Revista Brasileira de Ciência do Solo, Campinas, v. 16, p. 271-276, 1992.

AXE, L.; ANDERSON, P. R. Intraparticle diffusion of metal contaminants in amorphous oxide minerals. In: JENNE, E. A. Adsorption of metals by geomedia: variables, mechanisms, and model applications. San Diego : Academic, 1998. p. 193-200.

BARROW, N. J. The reaction of plants nutrients and pollutants with soils. II. Effect of $\mathrm{pH}$. Soil Science, Baltimore, v. 153, p. 195-204, 1989.

BARROW, N. J. Testing a mechanistic model IV. Describing the effect of $\mathrm{pH}$ on $\mathrm{Zn}$ retention by soils. Journal of Soil Science, Oxford, v. 37, p. 295-302, 1986.

CHLOPECKA, A.; BACON, J. R.; WILSON, M. J.; KAY, $\mathrm{J}$. Forms of cadmium, lead, and zinc in contaminated soils from southwest Poland. Journal of Environmental Quality, Madison, v. 25, p. 69-79, Jan./Feb. 1996.

CRAVO, M. S.; MURAOKA, T.; GINÉ, M. F. Caracterização química de compostos de lixo urbano de algumas usinas brasileiras. Revista Brasileira de Ciência do Solo, Campinas, v. 22, p. 547-553, 1998.

DAVIS, J. A.; LECKIE, J. O. Surface ionization and complexation at the oxide/water interface. Journal of Colloid and Interface Science, San Diego, v. 67, p. 91107, 1978.

GUILHERME, L. R. G.; ANDERSON, S. J. Copper sorption kinetics and sorption hysteresis in two oxiderich soils (Oxisols): effect of phosphate pretreatment. In: JENNE, E. A. Adsorption of metals by geomedia: variables, mechanisms, and model applications. San Diego : Academic, 1998. p. 209-228.
HARTER, R. D. Adsorption of copper and lead by Ap and B2 horizons of several Northeastern United States soils. Soil Science Society of America Journal, Madison, v. 43, p. 679-683, 1979.

HAYES, K. F.; LECKIE, J. O. Mechanism of lead ion adsorption at the goethite-water interface. In: DAVIS, J. A.; HAYES, K. F. Geochemical processes at mineral surfaces. Washington : American Chemical Society, 1986. p. 114-141.

KABATA PENDIAS, A.; PENDIAS, H. Trace elements in soils and plants. 2. ed. Boca Raton : CRC, 1992. 365 p.

LINDSAY, W. L. Chemical equilibria in soils. New York : Wiley-Interscience, 1979. 449 p.

McBRIDE, M. B. Environmental chemistry of soils. New York : Oxford University, 1994. 406 p.

McKENZIE, R. M. The adsorption of lead and other heavy metals on oxides of manganese and iron. Australian Journal of Soil Research, Collingwood, v. 18, p. 61-73, 1980.

MATOS, A. T. Fatores de retardamento e coeficientes de dispersão-difusão de zinco, cádmio, cobre e chumbo em solos do município de Viçosa. Viçosa : UFV, 1995. 110 p. Tese de Doutorado.

MATOS, A. T.; FONTES, M. P. F.; JORDÃO, C. P.; COSTA, L. M. Mobilidade e formas de retenção de metais pesados em Latossolo Vermelho-Amarelo. Revista Brasileira de Ciência do Solo, Campinas, v. 20, p. 379-386, 1996.

NAIDU, R.; BOLAN, N. S.; KOOKANA, R. S.; TILLER, $\mathrm{K}$. G. Ionic-strength and $\mathrm{pH}$ effects on the sorption of cadmium and the surface charge of soils. European Journal of Soil Science, Oxford, v. 45, p. 419-429, Dec. 1994.

SCHINDLER, P. W.; LIECHTI, P.; WESTALL, J. C. Adsorption of copper, cadmium, and lead from aqueous solution to the kaolinite/water interface. Netherlands Journal of Agricultural Science, Wageningen, v. 35, p. 219-230, 1987.

SILVA, M. L. N.; CURI, N.; MARQUES, J. J. G. S. M.; GUILHERME, L. R. G.; LIMA, J. M. Ponto de efeito salino nulo e suas relações com propriedades mineralógicas e químicas de latossolos brasileiros. Pesquisa Agropecuária Brasileira, Brasília, v. 31, n. 9, p. 663-671, set. 1996.

SPARK, K. M.; JOHNSON, B. B.; WELLS, J. D. Characterizing heavy-metal adsorption on oxides and oxyhydroxides. European Journal of Soil Science, Oxford, v. 46, p. 621-631, Dec. 1995. 
YU, T. R.; SUN, H. Y.; ZHANG, H. Specific adsorption of cations. In: YU, T. R. Chemistry of variable charge soils. New York : Oxford University Press, 1997. p. 140-174.

ZELAZNY, L. W.; HE, L.; VANWORMHOUDT, A. Charge analysis of soils and anion exchange. In: SPARKS,
D. L.; PAGE, A. L.; HELMKE, P. A.; LOEPPERT, R. H.; SOLTANPOUR, P. N.; TABATABAI, M. A.; JOHNSON, C. T.; SUMNER, M. E. (Ed.). Methods of soil analysis: chemical methods. Madison : Soil Science Society of America/American Society of Agronomy, 1996. pt. 3, p. 1231-1253. 